\title{
MEGAMASERS IN YOUNG ACTIVE GALACTIC NUCLEI
}

\author{
WILLEM A. BAAN \\ Arecibo Observatory \\ Arecibo, P.R. 00613
}

Megamaser emission provides new insights into the physics of active galactic nuclei. The megamaser phenomenon is consistent with a low-gain amplification model, where inverted foreground molecules amplify the radio continuum from the nucleus. The molecular gas seen in emission in the megamaser galaxies is located between 50 and 300 parsecs from the nucleus covering part of a possible NLR. Megamasers have been found for the molecules $\mathrm{OH}, \mathrm{H}_{2} \mathrm{CO}$ and $\mathrm{H}_{2} \mathrm{O}$. However, only $\mathrm{OH}$ megamasers form a clearly identifiable group of galaxies. They occur in prominent FIR galaxies and the FIR radiation field serves as a pumping agent for the $\mathrm{OH}$ molecules (Baan 1988). As a result only the ground state lines of $\mathrm{OH}$ are in emission, while the lines from excited states are in absorption (see Henkel, Güsten and Baan 1987).

The megamaser galaxies are congregated together in the FIR color-color diagrams and are part of the small population of extreme luminosity FIR (or ELF) galaxies. When the nuclear activity in these FIR galaxies tapers off and the dust around the nucleus is being consumed or blown away, these galaxies become less luminous and migrate across the FIR color-color diagrams into the region where the Seyferts and quasars lie. Therefore, the ELF galaxies and OH megamasers must have nuclear activity which has turned on only recently; they are young FIR nuclei (Baan, Henkel and Haschick 1987; Baan 1988; Sanders et al. 1988a,b).

A total of 18 megamasers have been found at present ranging in $\mathrm{OH}$ luminosity from 1 to $1000 \mathrm{~L}_{\odot}$. A comparison of the galaxies with $\mathrm{OH}$ emission and those with absorption ( 28 galaxies with absorption) shows that the megamasers occur in more luminous FIR galaxies than do the absorbers. Although both groups of $\mathrm{OH}$ galaxies have significant column densities along the line-of-sight to the nuclear radio source and could have a well-defined molecular disk, the FIR properties clearly separate the two groups. As the nuclear activity and the FIR luminosity decay with time, the $\mathrm{OH}$ emission will likely turn into absorption. The $\mathrm{OH}$ luminosity varies with the FIR luminosity as $\mathrm{L}_{O H} \propto \mathrm{L}_{I R}^{2}$. Such a dependence is consistent with a low-gain amplification picture, because $\mathrm{S}_{O H} \propto \tau_{O H} \mathrm{~S}_{18 \mathrm{~cm}}$ and both $\tau_{O H}$ and 402 
$\mathrm{S}_{18 \mathrm{~cm}}$ vary with the FIR flux (Baan 1989).

The $\mathrm{L}_{O H}-\mathrm{L}_{I R}$ relationship makes it possible to predict the number of detectable $\mathrm{OH}$ megamasers at a certain luminosity. This number increases as $\mathrm{L}_{O H}^{1 / 2}$ if the $\mathrm{OH}$ megamasers follow the observed FIR luminosity distribution at high $\mathrm{L}_{I R}$ (Lawrence et al. 1986; Soifer et al. 1987). The quadratic dependence of $\mathrm{L}_{O H}$ and $\mathrm{L}_{I R}$ makes it more probable to detect a far-away powerful maser than a weak nearby one. The fraction of $\mathrm{OH}$ megamasers among the FIR galaxies is found to increase with the FIR luminosity. For FIR sources with $\mathrm{L}_{I R}>10^{11} \mathrm{~L}_{\odot}$ about 20 percent of the sources show $\mathrm{OH}$ emission, while for sources above $10^{12} \mathrm{~L}_{\odot}$ more than 50 percent are masers. Twenty percent would be consistent with $\mathrm{OH}$ amplification occuring within a compact molecular disk but fifty is too high for a disk geometry. Since the amplification model only requires a sufficient column density towards the nucleus, it appears that the superluminous FIR sources are indeed the result of mergers and that the molecular gas is not confined to a disk (Baan 1989).

The $\mathrm{OH}$ emission originates in the direct neighborhood of an active nucleus. Superwinds eminating from the nucleus have been inferred from the extended optical and x-ray emission regions in some prominent active galaxies (Heckman, Armus and Miley 1987; Fabbiano and Trinchieri 1984). A superwind causes a blow-out along the symmetry axis of the galaxy, but it also interacts with the material in a molecular disk resulting in high outflow velocities. High velocity wings have been seen in some $\mathrm{OH}$ megamaser emission lines. The outflow velocity increases with the infrared luminosity of the galaxy, which is consistent with having the $\mathrm{OH}$ amplification occur close to the active nucleus. The molecular outflows become less pronounced as the nuclear luminosity decays and eventually a distinct expanding shell of swept-up gas may be formed; such shells are also observed in some $\mathrm{OH}$ absorbers and emitters (Baan, Haschick and Henkel 1988).

References.

Baan, W.A. 1988, Ap.J., 330, 743.

Baan, W.A. 1989, Ap.J., in the press.

Baan, W.A. and Haschick, A.D. and Henkel, C. 1988, Ap.J., submitted.

Baan, W.A., Henkel, C., and Haschick, A.D. 1987, Ap.J., 320, 154.

Fabbiano, G. and Trinchieri, G. 1984, Ap.J., 286, 491.

Heckman, T., Armus, L., and Miley, G. 1987, A.J., 92, 276.

Henkel, C., Güsten, R., and Baan, W.A. 1987, Astr.Ap., 185, 14.

Lawrence, A., et al. 1986, M.N.R.A.S., 219, 687.

Sanders, D., et al. 1988a, Ap.J., 325, 74.

Sanders, D., et al. 1988b, Ap.J., 328, L35.

Soifer, B.T., et al. 1987, Ap.J., 320, 238. 